\title{
Glottopol
}

Revue de sociolinguistique en ligne

$34 \mid 2020$

Les « langues de France ", 20 ans après

Jean Le Dû \& Yves Le Berre, Métamorphoses. Trente ans de sociolinguistique à Brest (1984-2014)

Brest, Centre de Recherche Bretonne et Celtique, 302 p., 2019.

\section{Salih Akin}

\section{(2) OpenEdition}

\section{Journals}

Édition électronique

URL : https://journals.openedition.org/glottopol/512

DOI : $10.4000 /$ glottopol.512

ISSN : 1769-7425

Éditeur

Presses universitaires de Rouen et du Havre

Référence électronique

Salih Akin, « Jean Le Dû \& Yves Le Berre, Métamorphoses. Trente ans de sociolinguistique à Brest (1984-2014) », Glottopol [En ligne], 34 | 2020, mis en ligne le 01 juillet 2020, consulté le 02 octobre 2021. URL : http://journals.openedition.org/glottopol/512; DOI : https://doi.org/10.4000/glottopol.512 


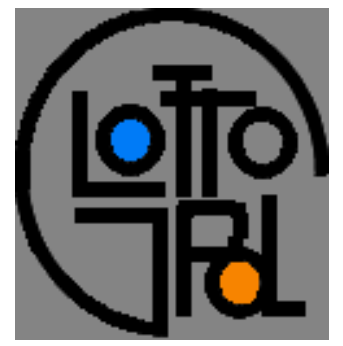

\section{GLOTTOPOL}

Revue de sociolinguistique en ligne $\mathrm{n}^{\circ} 34$ - juillet 2020

Les "langues de France " : 20 ans après

\section{SOMMAIRE}

Hommages à Jean Le Dî

Christian Lagarde : «Langues de France ». Au-delà du symbolique?

Entretien avec Bernard Cerquiglini, par Christian Lagarde.

Entretien avec Paul de Sinety, Délégué général à la langue française et aux langues de France, par Christian Lagarde.

Georg Kremnitz : La problématique initiale de la liste Cerquiglini et ses effets ultérieurs.

Alain Viaut : De "langue régionale » à "langue de France » ou les ombres du territoire.

Wanda Mastor: Le statut constitutionnel des langues régionales en droit comparé. De la reconnaissance à l'indifférence.

Philippe Martel, Marie-Jeanne Verny: Les langues régionales au Parlement, ou l'éternel retour.

Romain Colonna : Les "langues de France " : des langues non-étatiques au pays de l'Étatnation.

Hervé le Bihan : La langue bretonne : une visibilité toute en retenue.

Véronique Bertile : Les langues d'outre-mer : des langues de France? Approche juridique.

Jacques Vernaudon: Les langues polynésiennes et kanak, des "langues de France»en contexte de décolonisation.

Luc Biichlé : Qu'advient-il de l'arabe de France? Mise en perspective sociolinguistique...

Pascal Ottavi : L'épervier, la cage et le passereau.

Marielle Rispail : Le francique lorrain, langue de France? Réflexions et témoignages.

\section{Compte rendu de lecture}

Par Salih Akin : Jean Le Dû \& Yves Le Berre, Métamorphoses. Trente ans de sociolinguistique à Brest (1984-2014), Brest, Centre de Recherche Bretonne, 2019, 302 p. 


\title{
COMPTE RENDU
}

Jean Le Dû \& Yves Le Berre, 2019, Métamorphoses. Trente ans de sociolinguistique à Brest (1984-2014), Brest, Centre de Recherche Bretonne et Celtique, 302 p.

\author{
par Salih Akin
}

Laboratoire Dylis EA7474

Jean Le Dû et Yves Le Berre ont publié l'année dernière l'ouvrage Métamorphoses. Trente ans de sociolinguistique à Brest (1984-2014) qui consacre une trentaine d'années de recherches menées au Centre de Recherche Bretonne et Celtique de l'Université de Bretagne Occidentale sur les langues et la société en Bretagne. Jean Le Dû, dont nous avons appris avec grande tristesse la disparition le 6 mai 2020, était professeur émérite de celtique mais surtout l'un des fondateurs de la sociolinguistique du breton. Parallèlement à de nombreuses publications sous forme de monographies, d'articles et de volumes collectifs, dont il sera question ci-dessous, Jean Le Dû a publié le Nouvel Atlas linguistique de la Basse-Bretagne (2001), ouvrage en deux volumes qui étudie à travers 600 cartes la variation géographique de la langue bretonne aux plans phonétique, morphologique et lexical, avec des enquêtes commencées dès l'année 1969. Il a également confectionné Le trégorrois à Plougrescant. Dictionnaire breton français $(900$ p.), édité en 2012 aux éditions Emgleo Breiz. Yves Le Berre, avec qui Jean Le Dû a parcouru une grande partie de son parcours scientifique, est également professeur émérite de celtique et auteur de nombreuses publications consacrées à l'étude de la production écrite en breton. Avant de publier Métamorphoses, Jean Le Dû et Yves Le Berre ont aussi édité le Dictionnaire pratique français-breton en 1975 et Proverbes et Dictions de Basse-Bretagne en 1985.

L'ouvrage Métamorphoses se présente sous la forme d'un recueil de 302 pages regroupant des articles, chapitres d'ouvrage, actes de colloques. Il s'agit de publications individuelles ou cosignées issues de recherches et de réflexions communes sur la place et le rôle de la langue bretonne dans la société et entreprises au Centre de recherche bretonne et celtique. Il retrace les étapes importantes de la constitution de la sociolinguistique du breton dans le même mouvement qui a consacré la naissance de la sociolinguistique française. L'ouvrage est encadré par une introduction, une liste des travaux des auteurs, un index des noms propres et un index des notions et acronymes.

Au cours de leur carrière et de leurs recherches communes, les auteurs ont recensé en tout une quarantaine de publications, mais n'en ont retenu que vingt-deux, après les avoir épurées de redondances et répétitions, ce qui en fait un livre cohérent et permet une lecture suivie. Publiés et organisés dans l'ordre chronologique de leur parution, chaque texte est précédé d'une courte présentation de contextualisation qui mentionne la date et le lieu et le support de 
publication. Si cette organisation chronologique donne l'avantage de suivre le cheminement intellectuel et épistémologique et la lente maturation de la réflexion des auteurs, un regroupement des publications autour des grands axes de recherches aurait peut-être permis de mieux articuler cette impressionnante et foisonnante production scientifique.

Les vingt-deux textes de l'ouvrage proposent des analyses de la situation du breton, du français et des effets de leur contact en Bretagne. Aux yeux des auteurs, le terrain breton se présente comme une sorte de «laboratoire de langue» qui leur permet de percevoir et d'examiner des phénomènes de société ou de langage souvent invisibles à une vaste échelle, dans le monde extérieur. Le but est de proposer des concepts, des éléments de méthodes applicables à d'autres situations sociolinguistiques de minoration mettant en présence des langues de statut sociopolitique asymétrique.

D'ailleurs, le terme Métamorphoses, choisi pour nommer l'ouvrage, résulte lui-même d'une observation tirée du terrain breton. Si l'étymologie du mot (du grec metamorphosis) pose un " changement de forme », il permet aux auteurs surtout de pointer un paradoxe : alors que l'urbanisation, la médiatisation et le prolongement généralisé des études ont réduit considérablement la fréquence d'usage et la capacité d'échange des anciens parlers liés à la ruralité, les idiomes urbains ont au contraire connu un essor et une diversification inouïs. Bien que le breton fasse partie des langues en danger selon l'Atlas interactif UNESCO des langues en danger ${ }^{1}$, impulsées par les écoles Diwan et des jeunes générations, de nouvelles formes d'appropriation du breton ont émergé depuis une vingtaine d'années donnant à cette langue une plus grande visibilité dans l'espace public.

Les vingt-deux textes de Métamorphoses peuvent être répartis dans une dizaine d'axes de recherches en fonction des thématiques abordées. Le premier axe porte sur les langues et la société en Bretagne. Cet axe est celui qui constitue le terrain de recherches privilégié des auteurs et qui fédère le plus de textes $\left(n^{\circ} 7,8,12,14,18,19,21,22\right)$. Ensuite, vient l'axe d'études qui a porté les contacts de langues en Bretagne $\left(n^{\circ} 2,3,16\right)$. Des réflexions théoriques sont menées autour de quelques concepts centraux élaborés à partir des registres discrets du breton (registre paritaire, disparitaire, sphère privée et publique de la parole, le triptyque badumes standards normes) et développés et illustrés dans les textes $n^{\circ} 8,9,11,13$ et 17 . Les langues et la politique linguistique de France sont au centre des réflexions dans deux textes $\left(\mathrm{n}^{\circ} 1,5\right)$. La littérature de langue bretonne fait l'objet de développements dans les textes $n^{\circ} 6$ et 10 , tandis que la créativité lexicale en breton est abordée dans le texte $n^{\circ} 4$. La dénomination de la langue bretonne est discutée respectivement dans les textes $\mathrm{n}^{\circ} 15$ et 20 .

L'axe sur les langues et la société en Bretagne qui a nourri les réflexions des auteurs traite du présent et de l'avenir de la dialectologie dans une perspective sociolinguistique (texte $\mathrm{n}^{\circ} 7$ ), des langues et institutions en Bretagne (texte $n^{\circ} 8$ ), des langues et usages sociaux en BasseBretagne (texte $n^{\circ} 12$ ), de l'imaginaire linguistique (texte $n^{\circ} 14$ ) où les auteurs présentent le triptyque badumes, standards, normes. Le texte $\mathrm{n}^{\circ} 21$ illustre la lente érosion de l'usage et de la transmission du breton dans la commune de Plougrescant, située à la pointe extrême nord de la Bretagne. La situation de la langue bretonne dans la société régionale contemporaine vient en en quelque sorte en conclusion du recueil puisqu'il est le dernier texte $\left(\mathrm{n}^{\circ} 22\right)$. Partant des usages récents du breton, les auteurs pointent les interactions entre les registres : le néo-breton enseigné à l'école, mais très éloigné des usages par la nature des néologismes et le badume, marqué par une grande variation. Si le néo-breton semble heurter des parents et grands-parents, qui n'y reconnaissent pas leur langue maternelle, des maitres tentent d'adopter des traits du badume pour éviter un rejet brutal par les familles. Les échanges entre enseignants, journalistes, acteurs culturels débouchent sur ce que les auteurs appellent un «standard », qui contient à la fois des traits du badume, des néologismes transparents et des emprunts. Les auteurs voient dans ce

\footnotetext{
${ }^{1}$ http://www.unesco.org/languages-atlas/index.php?hl=fr\&page=atlasmap\&cc $2=\mathrm{FR}$
} 
standard, qui n'est pas rejeté par les vieux bretonnants, une sorte de voie du juste milieu qui joue le rôle de langue communautaire au sein d'une partie de la population.

Dans le domaine des contacts de langues en Bretagne, les auteurs dressent un état des lieux d'une part des parlers bretons en Bretagne et en Basse-Bretagne (texte $n^{\circ} 2$ ) et d'autre part le contact entre le français et le breton (texte $n^{\circ} 3$ ). La Bretagne souvent considérée comme une entité homogène du point de vue linguistique et culturelle, renferme selon les auteurs une hétérogénéité constitutive due à sa division au XVII ${ }^{\mathrm{e}}$ siècle en deux parties : la Haute-Bretagne qui correspond à la partie romane et la Basse-Bretagne qui est la partie bretonnante. Chacune de ces parties est loin d'être homogène. Les auteurs constatent en effet que les difficultés d'intercompréhension entre bretonnants de régions diverses sont liées à l'absence de contacts et augmentées par une ignorance quasi-totale de la langue écrite actuelle.

Le contact du breton avec le français vient amplifier l'hétérogénéité linguistique, puisque ce contact début très tôt, entre 1250 et 1280 , lorsque les bretonnants, en devançant l'institution, ont adopté le français comme langue des chartres en Basse-Bretagne. La diffusion du français s'amplifie au fur et à mesure que les Bretons intègrent la citoyenneté de la République en participant à la gestion des affaires locales, en accédant aux emplois civils et militaires de la fonction publique, ce qui débouchera sur leur scolarisation obligatoire en français à partir de la Révolution.

Mais cette diffusion entraine aussi une réaction pour la défense de la langue bretonne au sein de nombreux sanctuaires comme les zones rurales, les couches âgées de la population, les relations familiales et vicinales. Elle donne aussi naissance à des discours sur la relation du breton au français, qui se développe dans deux directions : d'une part l'affirmation de la spécificité, qui tendrait à se subdiviser à l'infini : la spécificité de la Bretagne en France, de la Basse-Bretagne en Bretagne, etc. Et d'autre part, la spécificité tend à s'universaliser en faisant apparaitre le combat breton comme une partie des combats des minorités en France et dans le monde.

Comme dans toute situation de contact mettant en présence des langues de statut sociopolitique asymétrique, le contact du breton avec le français entraine des conséquences dans le répertoire linguistique des populations bretonnantes. Les locuteurs naturels du breton connaissent presque tous le français et vivent une situation de diglossie déclinante, c'est-à-dire que chaque langue possède son propre champ d'exercice, le français tendant à envahir de l'extérieur et de l'intérieur le territoire du breton. Les locuteurs « culturels » du breton, ceux qui ont acquis le breton comme une L2, résistent aux influences du lexique français. Cette configuration voir apparaitre des variétés linguistiques intermédiaires comme le breton des francophones et le français des bretonnants. Alors que le premier jouit d'un certain prestige avec des supports institutionnels (livres, audio, télévision, enseignement), le second est considéré comme une simple déformation quand bien même il conserve beaucoup plus des traits spécifiques.

L'axe consacré aux langues et à la politique linguistique de France débute avec le texte $\mathrm{n}^{\circ} 1$ du recueil présenté au Premier Symposium international "Problèmes de glottopolitique », organisé à l'université de Rouen en 1984. Ce texte qui constitue un premier balisage du terrain breton décrit la lente régression de ses champs d'usage sous les effets de la politique linguistique de France. Les projets de loi en faveur des langues de France sous la IV ${ }^{\text {ème }}$ et la $\mathrm{V}^{\text {ème }}$ République sont abordés dans le texte $\mathrm{n}^{\circ} 5$. De la première proposition en faveur de l'introduction du breton dans l'enseignement déposé dans les années 1920 au rapport Deixonne de 1949, les auteurs discutent de la prise de conscience de l'importance de la préservation du breton dans la classe politique régionale et nationale, ainsi que des premiers pas réalisés vers l'introduction du breton dans l'enseignement comme dans les affichages publics bilingues.

Les concepts et notions théoriques élaborés à partir des registres discrets du breton sont discutés dans les textes $n^{\circ} 8,9,11,13$ et 17 . Ainsi, les registres de la parité et de la disparité qui 
traduisent les différents niveaux de langues selon la proximité ou la distance entre les interlocuteurs. Le registre de la parité est celui qui caractérise les interactions de l'intimité, de la fratrie, de la familiarité. Il s'agit d'un contexte où les participants de l'énonciation partagent plus ou moins le même profil sociologique et peuvent échanger de façon égalitaire. Le rayon de communication est géographiquement et socialement limité à l'oralité et s'il peut s'écrire, c'est plutôt de l'oral transcrit. Le registre de la disparité reflète les interactions marquées plus ou moins par le sceau de l'officialité et en conséquence un niveau formel de la langue. Les auteurs le considèrent comme le versant froid de la communication qui caractérise l'autorité, la représentativité, l'institution, etc. Le registre, basé sur l'écriture et destiné à une collectivité, bénéficie d'une capacité de communication universelle. Les auteurs donnent l'exemple d'un même contenu pour illustrer les deux registres: Tu m'ennuies (registre de la disparité), tu m'embêtes (registre de la parité). Ainsi, le registre paritaire relève de la sphère privée, alors que le registre disparitaire est de la sphère publique.

Le triptyque «badumes, standards, norme », qui a donné lieu à un colloque à l'Université de Brest en 1994, complexifie davantage l'opposition paritaire / disparitaire en intégrant l'idée d'un continuum des registres et une approche moins conflictuelle des situations de diglossie.

Préférant éviter l'usage des termes sociopolitiquement chargés comme dialecte, patois, langue, les auteurs mettent en circulation le terme badum, d'origine bretonne, signifiant «ce qu'on dit par ici ». Les badumes désignent des parlers dits populaires ou dialectaux et sont exempts de toute intervention institutionnelle. Ils ont pour principal caractère d'être un parler familier, identitaire, qui ne fait pas l'objet d'un enseignement mais d'une acquisition naturelle par imprégnation. C'est un registre qui est inscrit dans l'oralité et rattaché à une société paysanne. À une échelle un peu plus large, on trouve les standards qui correspondent aux formes écrites et codifiées de la langue destinées à la communication sur un territoire plus étendu que la paroisse ou le canton et sont pourvus d'institutions. Le standard gomme partiellement la variation des badumes dans le but de créer un espace de communication institutionnel. Enfin, la norme constitue l'étape ultime d'intégration des standards sous l'autorité d'un État national.

Dans les deux textes $\left(n^{\circ} 6,10\right)$ consacrés à la genèse de la littérature de langue bretonne, les auteurs analysent l'importance de l'écriture dans les mouvements militants et de défense de la langue bretonne. Selon les auteurs, cette littérature militante véhicule le thème d'une oppression exercée par la culture française sur la langue bretonne depuis le Moyen Âge jusqu'à nos jours, oppression qui serait à l'origine d'un recul continu des manifestations normales de la langue. Un schéma général d'une histoire de la littérature de langue bretonne est proposé que les auteurs présentent comme celui d'un processus d'acculturation cyclique de l'ensemble de la population celtophone de Bretagne. Chaque cycle concerne un groupe social en position d'accession à la culture écrite. Chaque groupe fait son entrée dans la sphère de l'écrit en adoptant une idéologie par laquelle il s'identifie. Un cycle commence par le passage de l'oralité à la lecture-écriture en breton. Cependant, à la fin du cycle, le groupe finit par écrire en français.

En étudiant la dénomination de la langue bretonne dans les textes $n^{\circ} 15$ et $n^{\circ} 20$, les auteurs montrent comment le nom de cette langue est un entrepôt de significations particulièrement denses. Langue des origines, langue pure, langue de la fidélité, langue pauvre (en termes intellectuels), langue riche (en termes dialectaux), le breton fait l'objet de catégorisations et représentations variant selon les milieux et les époques. Apparait alors l'importance de la dénomination des langues et des processus complexes qu'elle recouvre tant au niveau purement linguistique qu'au niveau politique et social.

Enfin, l'étude de la création lexicale en breton (texte $n^{\circ} 4$ ) montre les difficultés de néologie pour les langues minoritaires dépourvues d'institutions représentatives et des mediums comme l'école, les médias, État qui permettraient la diffusion des termes nouvellement créés. Alors que le breton manque de vocabulaire dans certains domaines techniques, juridiques, médicaux, 
etc., les auteurs constatent qu'aucun des néologismes créés au cours des deux derniers siècles n'est passé durablement dans la pratique langagière des bretonnants.

L'ensemble des textes est rédigé avec suffisamment de distance et de recul et révèle l'obstination de Jean Le Dû et d'Yves Le Berre à ne pas mêler recherche et militantisme, ce qui est une posture remarquable s'agissant des locuteurs-chercheurs d'une langue minorée en danger. Dans un entretien au quotidien Libération du 25 juin 1996, Jean Le Dû disait d'ailleurs que «Le breton, j'en ai fait le deuil au cours d'une longue thérapie mutuelle avec Yves Le Berre. Les nationalistes, eux, sont dans la mélancolie. Dans le deuil impossible. ». 


\section{GLOTTOPOL}

Revue de sociolinguistique en ligne

Comité de rédaction : Michaël Abecassis, Salih Akin, Sophie Babault, Claude Caitucoli, Véronique Castellotti, Régine Delamotte, Robert Fournier, Stéphanie Galligani, Emmanuelle Huver, Normand Labrie, Foued Laroussi, Benoit Leblanc, Fabienne Leconte, Gudrun Ledegen, Danièle Moore, Clara Mortamet, Alioune Ndao, Isabelle Pierozak, Gisèle Prignitz.

Rédactrice en chef : Clara Mortamet.

Comité scientifique : Claudine Bavoux, Michel Beniamino, Jacqueline Billiez, Philippe Blanchet, Pierre Bouchard, Ahmed Boukous, Pierre Dumont, Jean-Michel Eloy, Françoise Gadet, Monica Heller, Caroline Juilliard, Jean-Marie Klinkenberg, Jean Le Dû $(\dagger)$, Marinette Matthey, Jacques Maurais, Marie-Louise Moreau, Robert Nicolaï, Didier de Robillard, Paul Siblot, Claude Truchot, Daniel Véronique.

\section{Comité de lecture pour ce numéro :}

Salih Akin, Carmen Alén Garabato, Sophie Babault, Philippe Blanchet, Henri Boyer, Véronique Castellotti, Marisa Cavalli, Jean-François De Pietro, Didier de Robillard, Alain Di Meglio, Ksenija Djordjevic, Jean Michel Eloy, Pascale Erahrt, Véronique Fillol, Monica Heller, Robert Fournier, Normand Labrie, Hervé Lieutard, Jean Le Dû (†), Marinette Matthey.

http://glottopol.univ-rouen.fr

ISSN : 1769-7425 